\title{
Analysis of Additives in Polyethylene with Desorption Chemical Ionization/Tandem Mass Spectrometry
}

\author{
S. W. CHEN and G. R. HER* \\ Department of Chemistry, National Taiwan University, Taipei, Taiwan R. O. C
}

\begin{abstract}
Desorption chemical ionization with ammonia as the reagent gas in combination with tandem mass spectrometry is described for the analysis of additives in polyethylene. The additives were extracted with toluene and then analyzed directly by desorption chemical ionization and tandem mass spectrometry. With ammonia as the reagent gas, mass spectra of additives were found to contain intense pseudomolecular ions with very little fragmentation. In addition, low-molecular-weight polyethylene molecules extracted with the additives were barely detectable, and thus there was less likelihood of interference. Collision-induced dissociation of the pseudomolecular ions provided great confidence in the assignment of additives.
\end{abstract}

Index Heading: Analysis of polymer additives by tandem mass spectrometry.

\section{INTRODUCTION}

Various compounds are added to commercial polymers to prolong polymer lifetimes and enhance physicochemical properties. These polymer additives include antioxidants, ultraviolet stabilizers, lubricants, anti-static agents, thermal stabilizers, etc. Indirect analytical methods involving solvent extraction or supercritical fluid extraction of the additives from a polymer matrix followed by off- or on-line chromatographic separation and spectroscopic detection have been successfully developed and widely used. ${ }^{1-8}$ These methods are generally time consuming and limited in specificity. In recent years, mass spectrometry (MS), a highly sensitive and highly specific technique, has received considerable attention as an alternative technique for the analysis of polymer additives. $^{9-20}$ Earlier MS works focus on the use of EI, CI, and GC/MS in the analysis of additives. The major drawback to these methods is that they are limited to thermally stable and relatively volatile compounds and therefore are not suitable for many high-molecular-weight polymer additives. This - problem has now been largely overcome by the development of a series of soft ionization techniques, such as fast atom bombardment (FAB), field desorption (FD), laser desorption (LD), etc. $F A B,{ }^{10-13}$ $\mathrm{FD},{ }^{14} \mathrm{LD},{ }^{12,15,16}$ and secondary ion mass spectrometry ${ }^{17}$ all have shown their potential in the analysis of additives from solvent extract and/or from bulk polymeric material. Although FAB has a reputation as the most often used soft ionization method, Wilkins and co-workers ${ }^{12}$ have shown that $L D$ is superior to FAB in the analysis of polymer additives, mainly because polymer additives fragment extensively under FAB conditions. Another soft ionization technique, desorption chemical ionization (DCI), has also demonstrated its potential in the analysis

Received 27 November 1992.

* Author to whom correspondence should be sent.
TABLE 1. Trade name, molecular weight, and chemical name of polymer additives.

\begin{tabular}{|c|c|c|c|}
\hline No. & Trade name & MW & Chemical name \\
\hline 1 & Iragnox 245 & 586 & $\begin{array}{l}\text { Tri(ethyl glycol)bis-3-(3-t-butyl-4- } \\
\text { hydroxy-5-methylphe- } \\
\text { nyl)propionate }\end{array}$ \\
\hline 2 & Irganox 259 & 638 & $\begin{array}{l}\text { 1,6-Hexamethylene bis-(3,5-di- } t \text { - } \\
\text { butyl-4-hydroxyhydrocinnamate) }\end{array}$ \\
\hline 3 & Irganox 1010 & 1176 & $\begin{array}{l}\text { Pentaerythritol tetrakis [3-(3,5-di- } t \\
\text { butyl-4-hydroxyphenyl)pro-pion- } \\
\text { ate] }\end{array}$ \\
\hline 4 & Irganox 1024 & 552 & $\begin{array}{l}\mathrm{N}, \mathrm{N} \text {-bis[1-oxo-3-(3,5-di-tert-butyl- } \\
\text { 4-hydroxyphe- } \\
\text { nyl)propane]hydrazine }\end{array}$ \\
\hline 5 & Irganox 1076 & 530 & $\begin{array}{l}\text { Octyldecyl 3-(3,5-di-tert-butyl-4- } \\
\text { hydroxyphenyl)propionate }\end{array}$ \\
\hline 6 & Irganox 1098 & 636 & $\begin{array}{l}\mathrm{N}, \mathrm{N}^{\prime} \text {-hexamethylene bis }(3,5-\mathrm{di}-t- \\
\text { butyl-4-hydroxyhydrocinnamam- } \\
\text { ide) }\end{array}$ \\
\hline 7 & Irganox 3114 & 783 & $\begin{array}{l}\text { Tris(3,5-di- } t \text {-butyl-4-hydroxyben- } \\
\text { zyl)isocyanurate }\end{array}$ \\
\hline 8 & DLTDP & 514 & Dilauryl thiopropionate \\
\hline 9 & DSTDP & 682 & Distearyl thiopropionate \\
\hline 10 & Naugard 524 & $\begin{array}{l}646 \\
225\end{array}$ & $\begin{array}{l}\text { Tris(2,4-dk-t-butylphenyl) phos- } \\
\text { phite }\end{array}$ \\
\hline 11 & Tinuvin $\mathrm{P}$ & & $\begin{array}{l}\text { 2-(2-hydroxy- } 5 \text {-methylphenyl)- } 2 \mathrm{H}- \\
\text { benzotriazole }\end{array}$ \\
\hline 12 & Tinuvin 144 & 684 & $\begin{array}{l}\text { 2- } t \text {-Butyl-2-(4-hydroxy-3,5-di- } t \text {-bu- } \\
\text { tylbenzyl)[bis(methyl-2,2,6,6-tet- } \\
\text { ramethyl-4-piperidi- } \\
\text { nyl)]dipropionate }\end{array}$ \\
\hline 13 & Tinuvin 320 & 323 & $\begin{array}{l}\text { 2-(2-Hydroxy-3,5-di- } t \text {-butylphe- } \\
\text { nyl)-2H-benzotriazole }\end{array}$ \\
\hline 14 & Tinuvin 326 & 315 & $\begin{array}{l}\text { 2-(3-t-Butyl-2-hydroxy-5-methyl- } \\
\text { phenyl)-2H-5-chlorobenzotria- } \\
\text { zole }\end{array}$ \\
\hline 15 & Tinuvin 328 & 351 & $\begin{array}{l}\text { 2-(2-Hydroxy-3,5-di- } t \text {-amylphe- } \\
\text { nyl)-2H-benzotriazole }\end{array}$ \\
\hline 16 & Tinuvin 440 & 435 & $\begin{array}{l}\text { 8-Acetyl-3-dodecyl-7,7,9,9-tetra- } \\
\text { methyl-1,3,8-triazaspi- } \\
\text { ro(4,5)decane-2,4-dione }\end{array}$ \\
\hline 17 & Tinuvin 770 & 480 & $\begin{array}{l}\text { Bis( } 2,2,6,6 \text {-tetramethyl-4-piperidi- } \\
\text { nyl) sebacate }\end{array}$ \\
\hline 18 & Tinuvin 622 & $>3000$ & $\begin{array}{l}\text { Poly-(N-b-hydroxyethyl- } 2,2,6,6- \\
\text { tetramethyl-4-hydroxy-piperidyl } \\
\text { succinate) }\end{array}$ \\
\hline 19 & Chimassorb 944 & $>2500$ & $\begin{array}{l}\text { Poly-\{6-[1,1,3,3-tetramethylbutyl)- } \\
\text { imino]-1,3,5-triaine-2,4-diyl] }\{2- \\
\text { (2,2,6,6-tetramethylpiperidyl)- } \\
\text { imino] }\}\end{array}$ \\
\hline 20 & & 281 & \\
\hline 21 & DOP & 390 & Dioctyl phthalate \\
\hline 22 & DBP & 278 & Dibutyl phthalate \\
\hline
\end{tabular}

of thermally labile and nonvolatile molecules. ${ }^{18,19}$ The technique involves rapid sample heating followed by thermal desorption and ionization under CI conditions. Considering the overall ease of DCI operation, the capability of analyzing nonvolatile compounds, and the selectivity provided by choosing different reagent gases, 

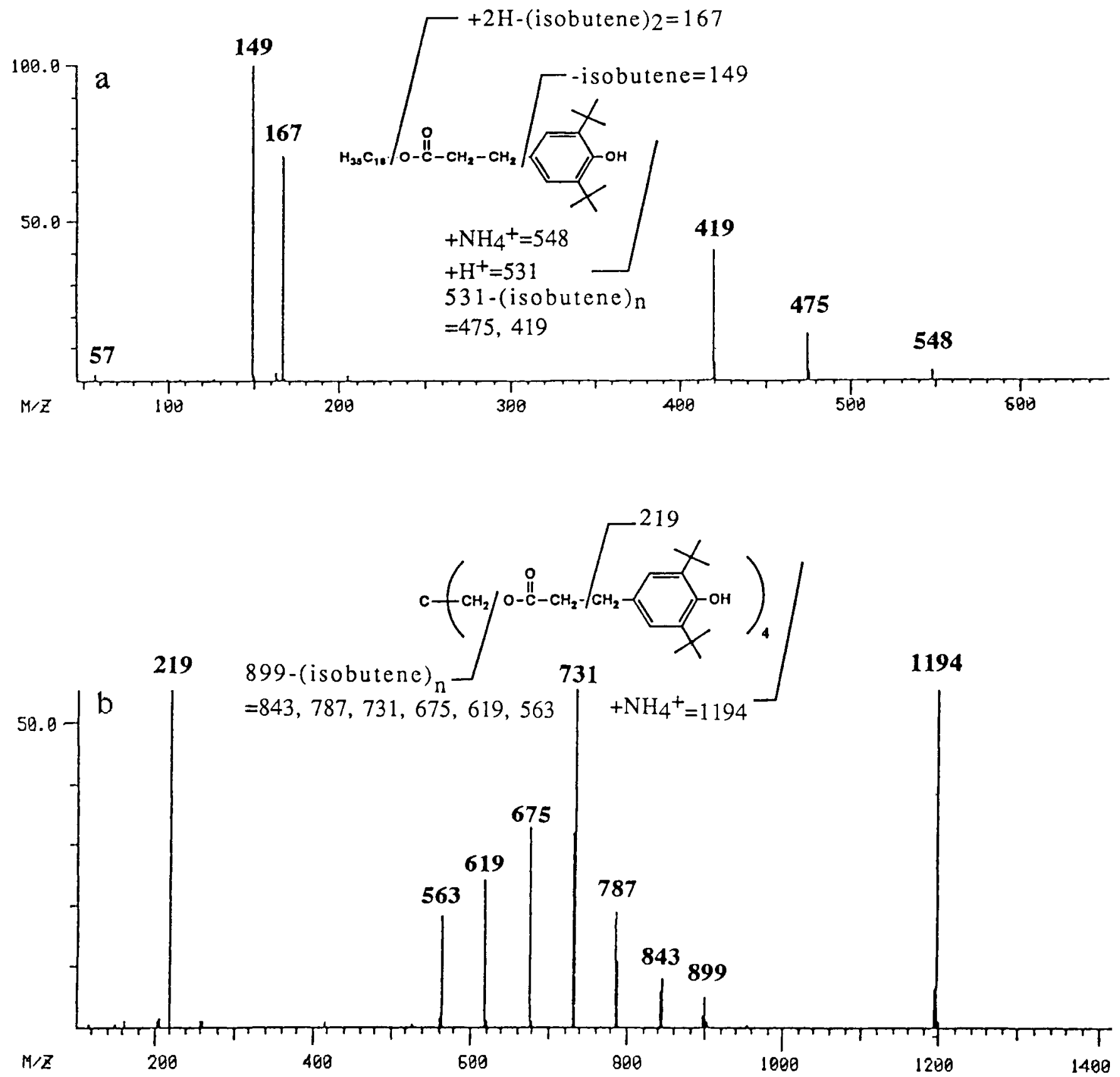

Fig. 1. CID mass spectrum of (a) $\left(\mathrm{M}+\mathrm{NH}_{4}\right)^{+}$ion $(m / z 548)$ of $\operatorname{Irganox} 1076 ;\left(\right.$ b) $\left(\mathrm{M}+\mathrm{NH}_{4}\right)^{+}$ion $(m / z 1194)$ of $\operatorname{Irganox} 1010$.

it is somewhat surprising that DCI has not been extensively used in the analysis of polymer additives.

Concurrent with the development of soft ionization techniques has been the development of tandem mass spectrometry (MS/MS). One of the major applications of MS/MS lies in the coupling with collision-induced dissociation (CID) in mixture analysis. In order to increase the sensitivity and also to facilitate the selection of a parent ion, a major criterion in the use of MS/MS in mixture analysis is that, upon ionization, each compound should produce as few ions as possible. Therefore, soft ionizations are often chosen in combination with tandem mass spectrometry in the analysis of mixtures. In this paper, we explore the potential of using ammonia DCI as the soft ionization technique along with tandem mass spectrometry in the analysis of additives from polyethylene extracts.

\section{EXPERIMENTAL}

Chemicals. Oleamide was purchased from TCI (Tokyo, Japan); all other additive standards were kindly provided by Ciba-Geigy, Ltd. (Taiwan). The trade names, molecular weights, and chemical names of the additive standards are listed in Table I. Oxidized Nugard 524 was prepared by the oxidation of Nugard 524 with hydrogen peroxide. The polyethylene samples were obtained from local stores.

Polymer Extracts. Approximately one gram of polymer sample was placed in a $250-\mathrm{mL}$ round-bottom bottle and 

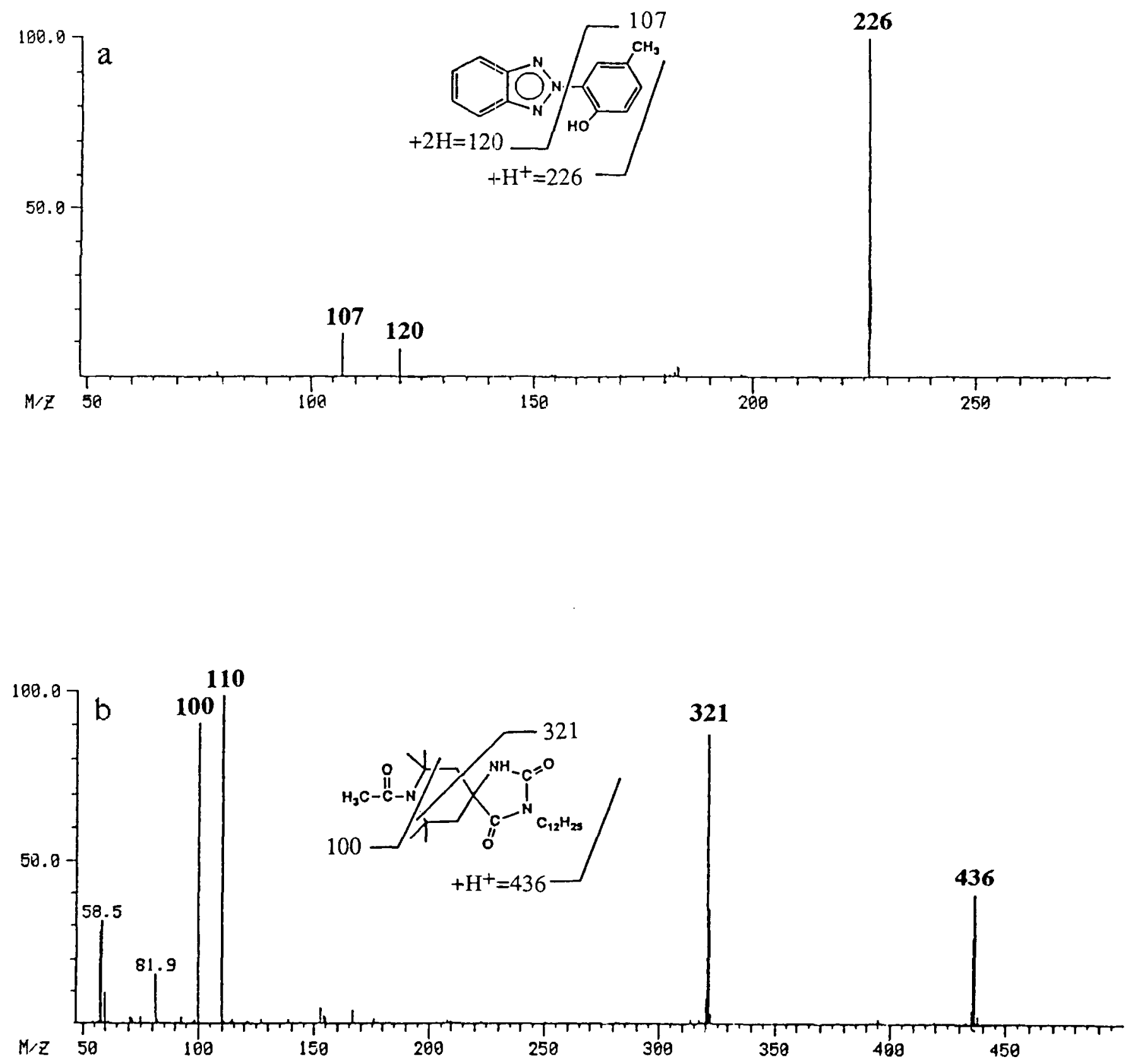

Fig. 2. CID mass spectrum of $(\mathbf{a})(\mathbf{M}+\mathbf{H})^{+}$ion $(m / z 226)$ of Tinuvin $\mathrm{P}$; $(\mathbf{b})(\mathbf{M}+\mathbf{H})^{+}$ion $(m / z 436)$ of Tinuvin 440 .

refluxed with $100 \mathrm{~mL}$ of toluene for 3 hours. After cooling, $20 \mathrm{~mL}$ of methanol was added to precipitate low-molecular-weight polymer. The liquid was filtered and dried. The residue was redissolved in methylene chloride and stored for subsequent analysis.

Mass Spectrometry. DCI/MS and DCI/MS/MS experiments were performed on a Finnigan TSQ-46C triplequadrupole mass spectrometer (Finnigan MAT, CA). Methane (from Matheson Co., NJ) and ammonia (from San-Fu Co., Hsinchu, Taiwan) were used as the reagent gas. The sample was air-dried on a platinum or rhenium emitter; the emitter was heated by a separated power supply at a heating rate of $10 \mathrm{~mA}$ per second or $20 \mathrm{~mA}$ per second until the maximum current of $1.3 \mathrm{~A}$ was reached. Argon was used as the collision gas.

\section{RESULTS AND DISCUSSION}

The selection of a suitable ionization method is important to the success of mixture analysis by MS/MS. Ideally, in order to minimize the interference in the selection of parent ions, only molecular ions should be produced for each of the compounds in the mixture. For this reason, the "softest" ionization technique is often the best choice in the analysis of mixtures with MS/MS. In addition to "softness," selectivity is also an important factor in the selection of the ionization technique. It is better to choose an ionization technique which responds preferentially to the analytes over the matrix because the polymer extract often consists of additives as well as a low-molecular-weight polymer "matrix." 

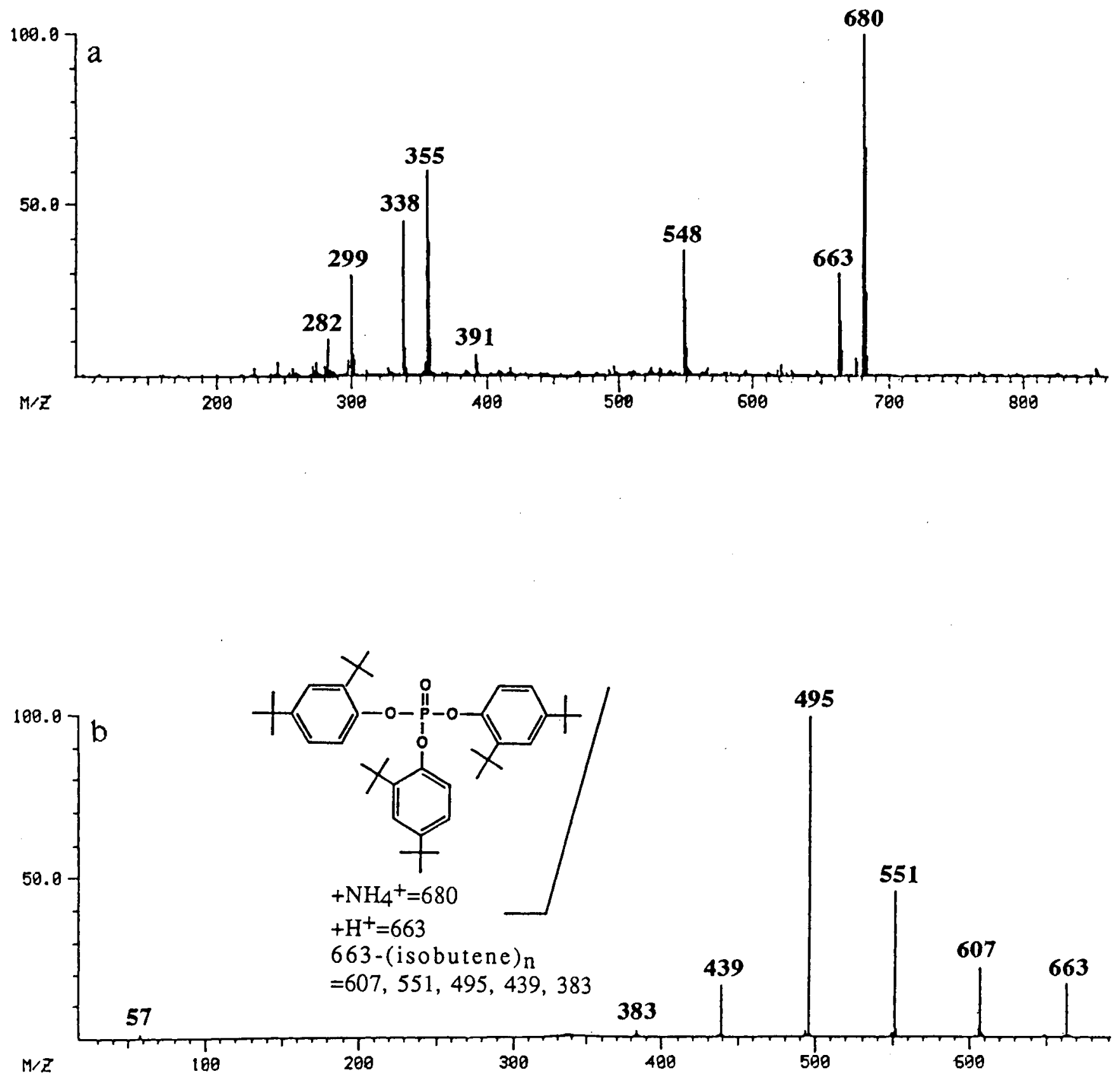

FIG. 3. (a) Ammonia DCI mass spectrum of a low-density polyethylene extract; (b) CID mass spectrum of the $\left(\mathbf{M}+\mathrm{NH}_{4}\right)+$ ion $(m / z$ 663) of oxidized Nugard 524.

When methane was chosen as the DCI reagent gas in the analysis of additive standards, pseudomolecular ion(s) as well as many fragment ions were observed. The spectra became more complicated when polyethylene extracts were analyzed directly by methane DCI. In addition to the molecular ions and many fragment ions from the additives, there were series of ions with a 14-amu interval corresponding to low-molecular-weight polyethylene molecules. These matrix ions may obscure ions from additives and make the assignment and the selection of precursor ions for MS/MS difficult.

Because of its high proton affinity, ammonia frequently gives very simple CI mass spectra consisting of pseudomolecular ions with very little fragmentation. Except for Tinuvin 622 and Chimassorb 944, with molecular weights beyond the mass range of the instrument used $(1800 \mathrm{amu})$, the ammonia DCI mass spectra of all the polymer additives investigated were very simple. The mass spectra were characterized with $(\mathrm{M}+\mathrm{H})^{+}$and/or $\left(\mathrm{M}+\mathrm{NH}_{4}\right)^{+}$ions and with very little fragmentation. Most likely because of the difference in proton affinity, the intensity of the $\left(\mathrm{M}+\mathrm{NH}_{4}\right)^{+}$adduct ion was higher than the $(\mathrm{M}+\mathrm{H})^{+}$ion for most phenolic antioxidants, whereas the intensity of the $(\mathrm{M}+\mathrm{H})+$ was significantly higher than the $\left(\mathrm{M}+\mathrm{NH}_{4}\right)^{+}$ion for Tinuvin light stabilizer. In addition to being "soft," ammonia is also considered a selective ionization technique. This property can be an advantage if the additives can be ionized preferentially over the matrix. ${ }^{20}$ The high proton affinity of ammonia makes the low-proton-affinity polyethylene 

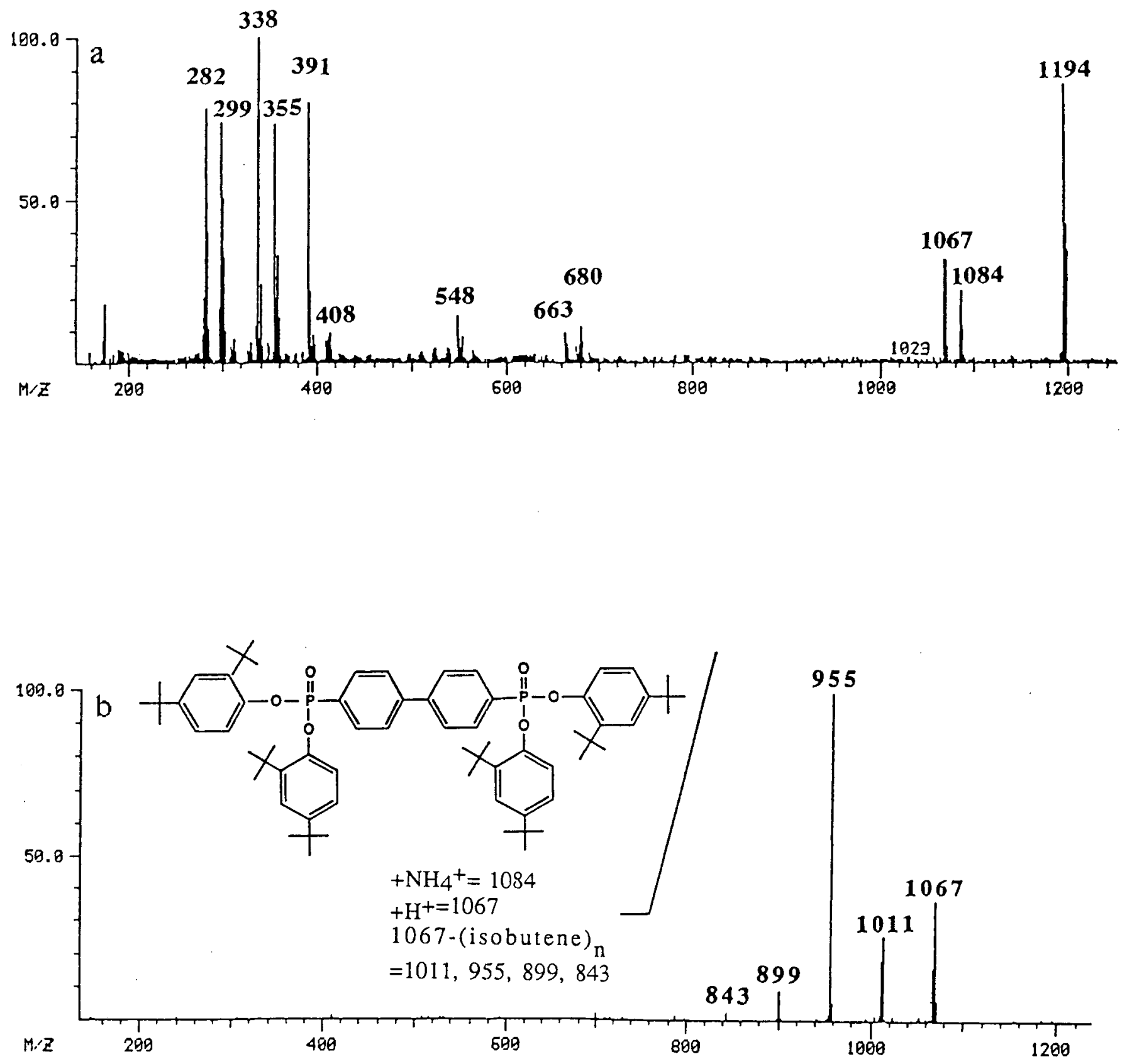

FIg. 4. (a) Ammonia DCI mass spectrum of a second low-density polyethylene extract; (b) CID mass spectrum of the $\mathrm{m} / z 1067$ ion.

matrix barely detectable, and thus there is less likelihood of interference in the analysis of additives. The lowmolecular-weight polyethylene ions, which were quite intense under the conditions of methane DCI, disappeared almost completely in ammonia DCI.

The ratio of the $(\mathrm{M}+\mathrm{H})^{+}$and $\left(\mathrm{M}+\mathrm{NH}_{4}\right)^{+}$ions provides some indication of the proton affinity of the analyte. Therefore, if reference compounds are available, it is possible to identify additives on the basis of the molecular weights and their $(\mathrm{M}+\mathrm{H})^{+} /\left(\mathrm{M}+\mathrm{NH}_{4}\right)^{+}$ ratios. Much greater specificity, however, can be obtained with the use of tandem mass spectrometry; the product (daughter) ion mass spectrum obtained with a tandem mass spectrometer provides many structurally characteristic fragment ions and thus much greater confidence in the assignment. The structures and the CID mass spectra of several common polymer additives are shown in Figs. 1 and 2. It is noticed that one of the common fragmentation pathways observed in the CID spectra of antioxidant is the loss of alkene(s) from the parent ions. For example, the fragments at $\mathrm{m} / z 475$ and 419 in Fig. 1a were produced because of the loss of one or two isobutenes from the ammoniated molecular ion $(\mathrm{m} / z$ 548) of Irganox 1076. A similar phenomenon was observed for Irganox 1010; a sequential loss of six isobutenes was recorded (Fig. 1b). Unlike the case for other UV stabilizers, the major fragment of Tinuvin 440 was produced due to the two-bond ring cleavage (double cleavage) reaction (Fig. 2b).

In an effort to evaluate the utility of ammonia DCI/ MS/MS for detecting polymer additives in polyethylene, several commercial polyethylene products were extract- 

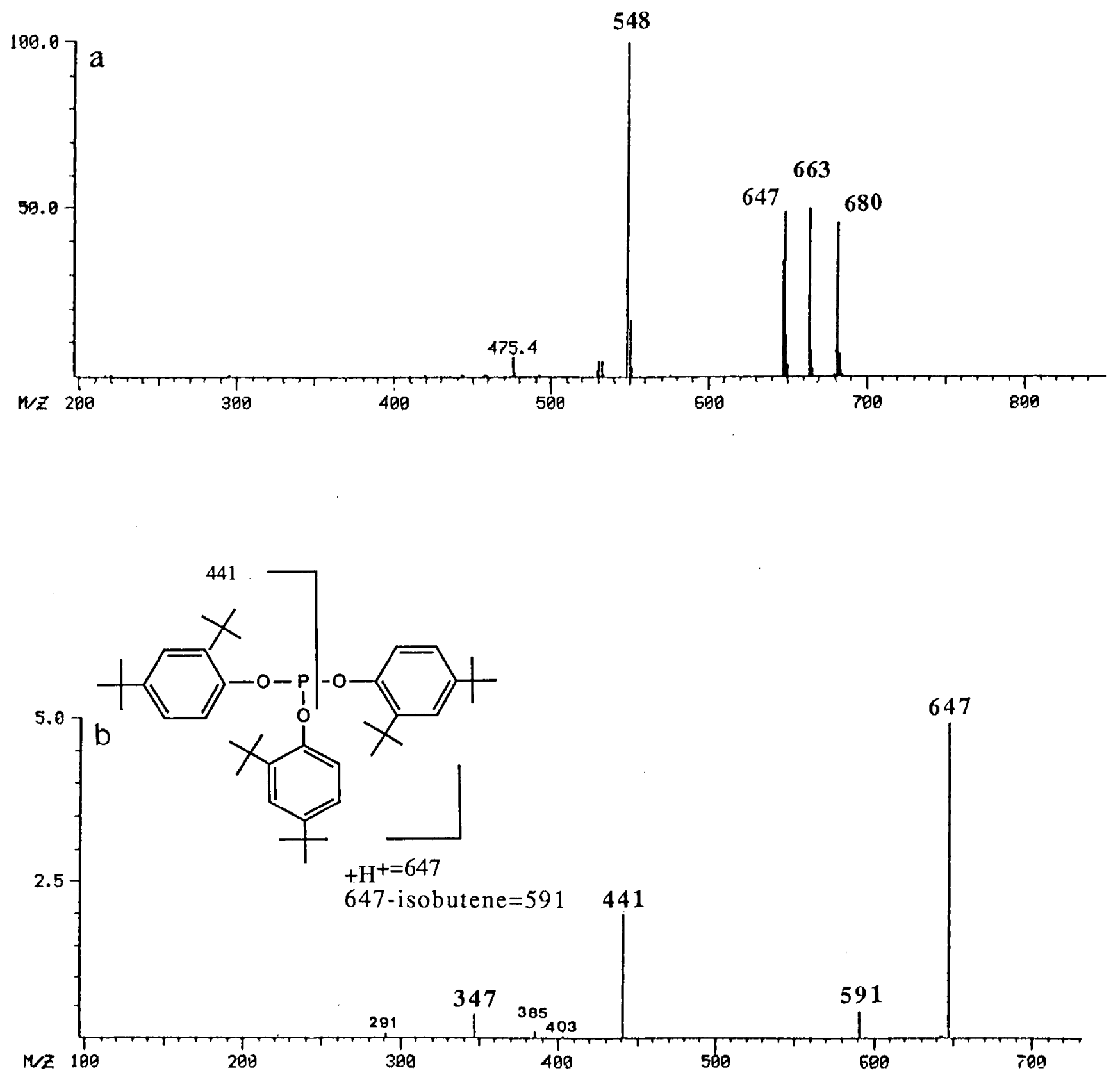

FIG. 5. (a) Ammonia DCI mass spectrum of a high-density polyethylene extract; (b) CID mass spectrum of the $\mathrm{m} / z 647$ ion.

ed with toluene and then analyzed directly by DCI/MS and DCI/MS/MS. The results of two low-density and two high-density polyethylene samples are presented. The ammonia DCI mass spectrum of a tarp sample which is used locally as a disposable raincoat is shown in Fig. 3a. Pseudomolecular ions for several additives are clearly distinguishable. The $(\mathrm{M}+\mathrm{H})+/\left(\mathrm{M}+\mathrm{NH}_{4}\right)^{+}$ion pairs at $m / z 282 / 299,338 / 355$, and $663 / 680$ correspond to additives with molecular weights of 281,337 , and 662 , respectively. The CID mass spectra of the $282 / 299$ and 338 / 355 ion pairs suggested that they were amide waxes oleamide and erucamide, respectively. The CID spectrum of the 663 ion is shown in Fig. 3b. This spectrum is very similar to not the intact form, but the oxidation form, of the phosphite triester secondary antioxidant, Naugard $524(\mathrm{MW}=646)$. The ion at $\mathrm{m} / z 548$ was observed in many samples. This ion is either the $(\mathrm{M}+\mathrm{H})^{+}$ ion for an additive with molecular weight of 547 or the $\left(\mathrm{M}+\mathrm{NH}_{4}\right)^{+}$ion for an additive with molecular weight of 530. It is assigned as the $\left(\mathrm{M}+\mathrm{NH}_{4}\right)^{+}$ion of the antioxidant Irganox 1076 because the CID spectrum is very similar to the spectrum of Fig. 1a. On the basis of the product ions $(m / z 149,167,279)$, the $m / z 391$ ion was assigned as the $(\mathrm{M}+\mathrm{H})^{+}$ion of the additive dioctyl phthalate (DOP). This compound has been used as a plasticizer in polyvinyl chloride or as a lubricant in lowdensity polyethylene. ${ }^{21}$ Because DOP is a common contaminant in samples that have been in touch with plastic or rubber materials, a blank test is used to eliminate the possibility of contamination.

More additives were detected for a disposable raincoat from another manufacturer. The DCI mass spectrum of this second tarp extract is shown in Fig. 4a. In addition to the additives identified in the first tarp extract, two 

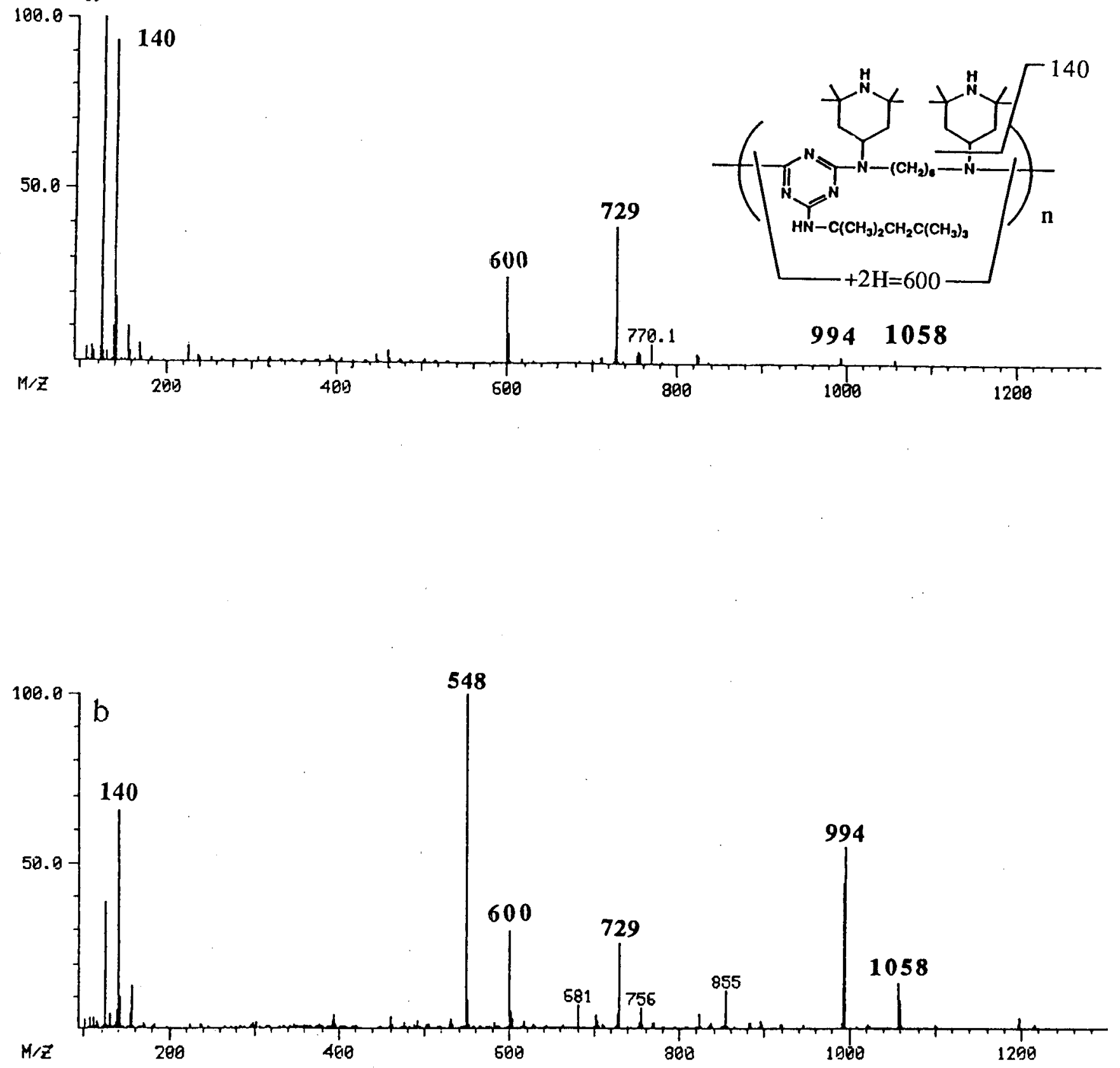

Fig. 6. (a) Ammonia DCI mass spectrum of Chimassorb 944; (b) ammonia DCI mass spectrum of a high-density polyethylene extract.

more additives with molecular weights of over $1000 \mathrm{amu}$ were detected and identified. The ions at $\mathrm{m} / z 1067$ and 1084 correspond to a compound with a molecular weight of 1066. Because the fragmentation pattern of the $\mathrm{m} / \mathrm{z}$ 1067 ion was similar to the oxidized Nugard 524 with sequential loss of isobutene from the parent ion (Fig. 4b), this ion was tentatively assigned as the $(\mathrm{M}+\mathrm{H})^{+}$ion of the compound resulting from the oxidation of the phosphite triester secondary antioxidant, Santostab PEPQ $(M W=1034)$. The ion at $m / z 1194$ was assigned as the $\left(\mathrm{M}+\mathrm{NH}_{4}\right)+$ ion of another common antioxidant, Irganox 1010, because the CID spectrum of the $m / z 1194$ ion was very similar to the spectrum in Fig. $1 \mathrm{~b}$.

Phosphite antioxidant Nugard 524 was also detected in a high-density sample (Fig. 5a). In addition to the pseudomolecular ions $(m / z 663,680)$ corresponding to the oxidized Nugard 524, intact Nugard $524(\mathrm{~m} / z$ 647) was also observed. It is interesting to see that an intact molecule has higher proton affinity than its oxidation product [the protonated molecular ion $(\mathrm{M}+\mathrm{H})^{+}$is the major pseudomolecular ion for Nugard 524, whereas (M $+\mathrm{H})^{+}$and $\left(\mathrm{M}+\mathrm{NH}_{4}\right)^{+}$have similar intensity for oxidized Nugard 524]. The difference in proton affinity is more likely due to the electron withdrawing effect of the oxygen atom. The CID spectra of the intact Nugard 524 (Fig. 5b) and oxidized Nugard 524 (Fig. 3b) show that oxidation also affects the fragmentation pattern significantly. The ion at $m / z 548$ is the ammoniated molecular ion of the antioxidant Irganox 1076.

In recent years, there has been a tendency to use stabilizer with higher molecular weight $(>2000 \mathrm{amu})$ to prevent loss under severe conditions of applications. 
When hindered amine light stabilizer Chimassorb 944 was studied by ammonia DCI, the molecular ion was not detected, probably because of the limited mass range of the mass spectrometer. However, many characteristic fragments were observed (Fig. 6a). These ions are very useful in the identification of additives. In the analysis of the extract from a high-density sample (beer crate), when the temperature of the DCI probe approached its maximum value, many high mass fragments were detected (Fig. 6b). Chimassorb 944 is believed to be present in this sample because (1) the fragments at $m / z 600,729$, 994 , and 1058 are the major fragments of Chimassorb 944 ; (2) the $m / z 600$ ion corresponds to the molecular weight of monomer; and (3) the $\mathrm{m} / z 140$ ion, observed in the CID of the $m / z 600$, is a structurally characteristic cyclic amine fragment ion. In addition to the UV absorber, antioxidant Irganox 1076 was also detected in this sample $(m / z 548$ ion in Fig. $6 \mathrm{~b})$.

\section{CONCLUSION}

The results show that ammonia $\mathrm{DCI} /$ tandem mass spectrometry is a convenient method for the detection of additives in polyethylene samples. The softness and selectivity provided by ammonia DCI in combination with the specificity provided by collision-induced dissociation demonstrate its great potential for identification of additives directly from polyethylene extracts. Knowing the amount of additives is at least as important as identifying the additives in the polymer sample; further study will explore the utility of DCI in the quantitative analysis of additives in polyethylene.

\section{ACKNOWLEDGMENT}

This work was supported by the National Research Council of the Republic of China.

1. J. F. Schabron and L. E. Fenska, Anal. Chem. 52, 1411 (1980).

2. R. B. Walter and J. F. Johnson, J. Polym. Sci. 15, 29 (1980).

3. D. Munteanu, A. Isfan, C. Isfan, and I. Tincul, Chromatographia 23, 7 (1987).

4. M. W. Raynor, K. D. Bartle, I. L. Davies, A. Williams, and A. A. Clifford, Anal. Chem. 60, 427 (1988).

5. C. Grosset, D. Cantin, A. Villet, and J. Alary, Talanta 37, 301 (1990).

6. R. C. Wieboldt, K. D. Kempfert, and D. L. Dalrymple, Appl. Spectrosc. 44, 1028 (1990).

7. M. Ashraf-Khorassani and J. M. Levy, J. High Resol. Chromatogr. 13, $742(1990)$.

8. B. Marcato, C. Fantazzini, and F. Sevini, J. Chromatogr. 553, 415 (1991).

9. R. P. Lattimer and R. E. Harris, Mass Spectrom. Rev. 4, 369 (1985).

10. J. O. Lay, Jr., and B. J. Miller, Anal. Chem. 59, 1323A (1987).

11. H. S. Freeman, Z. Hao, J. Sokolowska-Gajda, R. B. van Breeman, and J. C. Le, Dyes and Pigments 16, 317 (1991).

12. C. L. Johlman, C. L. Wilkins, J. D. Hogan, T. L. Donovan, D. A. Laude, Jr., and M. J. Youssefi, Anal. Chem. 62, 1167 (1990).

13. T. L. Riley, T. J. Prater, J. L. Gerlock, J. E. de Vries, and D. Schuetzle, Anal. Chem. 56, 2145 (1984).

14. R. P. Lattimer, R. E. Harris, D. B. Ross, and H. E. Diem, Rubber Chem. Technol. 57, 1013 (1984).

15. A. T. Hsu and A. G. Marshall, Anal. Chem. 60, 932 (1988).

16. B. Asamoto, J. R. Young, and R. J. Citerin, Anal. Chem. 62, 61 (1990).

17. M. P. Mawn, R. W. Linton, S. R. Bryan, B. Hagenhoff, U. Jurgens, and A. Benninghoven, J. Vac. Sci. Technol. A 93, 1307 (1991).

18. R. J. Cotter, Anal. Chem. 52, 1589A (1980).

19. M. Vincenti, E. Pelizzetti, A. Guarini, and S. Costanzi, Anal Chem. 64, 1879 (1992).

20. P. Rudewicz and B. Munson, Anal. Chem. 58, 358 (1986).

21. R. F. Grossman, "Lubricants," in Thermoplastic Polymer Additives, J. T. Lutz, Jr., Ed. (Marcel Dekker, New York, 1989), Chap. 7, p. 282. 